\title{
Nutzen-Risiko-Bewertung neuer Wirkstoffe als Grundlage für Priorisierungsentscheidungen in der Hämatologie/Onkologie: Methodische Herausforderungen und Lösungsansätze*
}

\author{
Wolf-Dieter Ludwig ${ }^{a, b} \quad$ Jan Schildmann ${ }^{c}$ \\ ${ }^{\text {a }}$ Klinik für Hämatologie, Onkologie und Tumorimmunologie, HELIOS Klinikum Berlin-Buch, \\ ${ }^{\mathrm{b}}$ Arzneimittelkommission der deutschen Ärzteschaft (AkdÄ), Berlin, \\ ${ }^{c}$ Institut für Medizinische Ethik und Geschichte der Medizin, Ruhr-Universität Bochum, Germany
}

\section{Schlüsselwörter \\ Spezialpräparate in der Onkologie . \\ Wirksamkeit/Risiko-Verhältnis · Zusatznutzen . \\ Kosten-Nutzen-Bewertung}

\section{Zusammenfassung}

Medikamentöse Therapieinnovationen in der Onkologie werfen medizinische und ethische Fragestellungen auf. Die zunehmende Inzidenz und Prävalenz von Krebserkrankungen sowie die Zulassung neuer, in aller Regel kostspieliger Wirkstoffe sind mit steigenden Gesamtkosten für die Behandlung von Krebserkrankungen verbunden. Deshalb ist die Entwicklung von wissenschaftlich begründeten Strategien zur Nutzenbewertung neuer medikamentöser Therapieverfahren unentbehrlich. Im Mittelpunkt dieses Beitrags steht die Nutzen- beziehungsweise Nutzen-RisikoBewertung als Grundlage für Entscheidungen über die Priorisierung bei hämatologischen Neoplasien und soliden Tumoren. Zunächst werden regulatorische Anforderungen an die Zulassung und Defizite in klinischen Zulassungsstudien als Grund für die zum Zeitpunkt des Markteintritts häufig nicht mögliche Nutzenbewertung neuer Wirkstoffe in der Onkologie dargestellt. Anschließend werden mögliche Lösungsansätze zur Optimierung einer Nutzen- bzw. Kosten-Nutzen-Bewertung und Vorschläge zur Verbesserung der wissenschaftlichen Datenlage diskutiert.

*Teile dieser Arbeit sind der Publikation Ludwig W-D, Fetscher S, Schildmann J: Teure Innovationen in der Onkologie - für alle? Überlegungen zu Voraussetzungen für eine rationale Pharmakotherapie und ethische Herausforderungen. Der Onkologe 2009;15:1004-1014 entnommen. Mit freundlicher Genehmigung von Springer Science and Business Media.

\section{Keywords \\ Specialty pharmaceuticals in oncology . \\ Benefit/risk balance $\cdot$ Added value of innovative oncology drugs · Cost-effectiveness analysis}

\begin{abstract}
Benefit-Risk Evaluation of New Drugs as a Basis for Decisions on Prioritization in Hematology/Oncology: Methodical Challenges and Problem-Solving Strategies Innovations in the drug treatment of cancer patients pose several medical and ethical challenges. The increasing incidence and prevalence of cancer, in combination with the availability of new and usually highly expensive anticancer drugs, are associated with a significant increase in the general costs for the treatment of cancer patients. Therefore, the development of scientific strategies for judgments on benefits is indispensable. In this paper, the authors analyze the benefit assessment and the benefitrisk assessment as a foundation for decisions on prioritization in hematology and oncology. In a first step, regulatory aspects and shortcomings regarding the design of clinical trials in oncology before and after approval of anticancer drugs are identified as factors that contribute to difficulties in establishing the effectiveness of new drugs entering the health care market. The authors will conclude with suggestions for the improvement of benefit-risk analyses and the generation of scientific data necessary for such analyses.
\end{abstract}

Prof. Dr. med. Wolf-Dieter Ludwig

Klinik für Hämatologie, Onkologie und Tumorimmunologie HELIOS Klinikum Berlin-Buch

Schwanebecker Chaussee 50, 13125 Berlin, Germany

Tel. +49 30 9401-52100, Fax -52109

wolf-dieter.ludwig@helios-kliniken.de 


\section{Einleitung}

Nutzen- bzw. Kosten-Nutzen-Bewertungen kostenintensiver neuer Therapieverfahren in der Hämatologie/Onkologie rücken zunehmend in den Blickpunkt der klinischen Versorgung sowie wissenschaftlicher und politischer Überlegungen [1-3]. Die pharmazeutische Industrie hat in den letzten Jahren eine strategische Neuausrichtung ihrer Forschungsaktivitäten mit Fokussierung auf die Hämatologie/Onkologie, aber auch HIV-Infektionen, chronische entzündliche und Zentralnervensystem (ZNS)-Erkrankungen (z.B. Psychosen und Alzheimer-Erkrankung) vorgenommen [4-6]. Angesichts der demographischen Entwicklung, deutlicher Fortschritte in der Grundlagenforschung mit der daraus resultierenden Entwicklung zahlreicher neuer Wirkstoffe mit zum Teil innovativen Angriffspunkten und des großen Bedarfes an Therapieverbesserungen in der Hämatologie/Onkologie ist dies grundsätzlich zu begrüßen. Mit Blick auf die Umsätze der pharmazeutischen Industrie fällt auf, dass sogenannte Blockbuster (Jahresumsatz > 1 Milliarde US Dollar) zunehmend durch sogenannte Nichebuster ersetzt wurden, die nach Zulassung als Arzneimittel für seltene Krankheiten (orphan drugs) häufig zunächst auch Off-Label eingesetzt werden und rasch eine Ausweitung ihrer Anwendungsgebiete anstreben [6-8]. Als Gründe für diese strategische Neuausrichtung werden genannt: Marktsättigung (welche Arzneimittel fehlen uns eigentlich?), Entwicklungskosten und Investitionsrisiken für breite Indikationsbereiche (z.B. Kardiologie), ein höhere Gewinne versprechender kommerzieller Markt und Patientenbedürfnisse (z.B. in der Onkologie), die noch nicht durch existierende Therapien abgedeckt sind. Der äußerst attraktive Arzneimittelmarkt in der Onkologie, mit derzeit jährlichen Wachstumsraten von 20-25\%, umfasst sowohl supportive Arzneimittel (z.B. Antiemetika, Antimykotika, hämatopoietische Wachstumsfaktoren) als auch zielgerichtete Wirkstoffe (z.B. Tyrosinkinase-Inhibitoren, monoklonale Antikörper). Interessanterweise spielen dabei die klassischen Zytostatika ökonomisch und daher vielfach auch in der klinischen Forschung eine immer kleinere Rolle [1, 2, 4, 6, 9].

Angesichts dieser Entwicklung und der daraus resultierenden enormen finanziellen Belastung für die Gesetzliche und Private Krankenversicherung (GKV, PKV) werden zunehmend Strategien gesucht, die eine effiziente Arzneimittelversorgung entsprechend dem aktuellen Stand des medizinischen Wissens in der Onkologie gewährleisten $[1,2,10]$. Dabei sollten der medizinische Fortschritt ebenso wie ethische Fragen, etwa in Bezug auf den Zugang zu medizinischen Leistungen und die gerechte Verteilung begrenzter Mittel im Gesundheitswesen, gleichermaßen berücksichtigt werden ([11], siehe auch Beitrag von E. Winkler in diesem Supplement). Im Folgenden werden ausgewählte methodische Herausforderungen der Nutzen- bzw. Nutzen-Risiko-Bewertung neuer Wirkstoffe in der Hämatologie/Onkologie sowie Strategien zur Implementierung der Nutzen-Risiko-Bewertung als Voraussetzung für eine angemessene Rationalisierung und Priorisierung in der Hämatologie/Onkologie dargestellt [2, 12, 13].

\section{Regulatorische Vorgaben und Defizite klinischer Zulassungsstudien: Relevanz für die Nutzen-Risiko- Bewertung bei neuen Arzneimitteln in der Hämatologie/Onkologie}

Die für neu zugelassene Wirkstoffe in der Hämatologie/Onkologie bei Einführung in die Gesundheitsversorgung verfügbaren Daten zur Wirksamkeit und Sicherheit sind heute vielfach unbefriedigend, da sie in der Regel keine abschließenden Aussagen zum Nutzen neuer Arzneimittel unter Alltagsbedingen («effectiveness») erlauben $[10,16,17]$. Verantwortlich hierfür sind neben den nicht immer konsequent beachteten und umgesetzten regulatorischen Vorgaben für klinische Studien insbesondere das Interesse der pharmazeutischen Hersteller an rascher Markteinführung ihrer neuen Wirkstoffe und das Bedürfnis der Patienten nach therapeutischen Alternativen für ihre häufig lebensbedrohlichen Erkrankungen [10, 18].

In diesem Beitrag wird zwischen der «Wirksamkeit» («efficacy») von Arzneimitteln und deren «Nutzen» («effectiveness») unterschieden. Die Wirksamkeit wird im Rahmen der Zulassungsstudien geprüft. Der Nutzen wird hier als das Ergebnis einer bewertenden Abwägung zwischen positiven und negativen Effekten unter Alltagsbedingungen im Hinblick auf die Indikation definiert. Die Bestimmung des Nutzens und die des Zusatznutzens im Vergleich zum geltenden Standard der Behandlung sind in der Regel erst nach der Zulassung möglich. Auf der Basis dieser Bewertung und unter Berücksichtigung der Kosten ist dann auch erst eine Bewertung des «Kosten/Nutzen-Verhältnisses» («efficiency») möglich [14, 15].

In den für die Zulassung besonders relevanten Phase-IIIStudien soll das Wirksamkeits-Risiko-Profil eines Arzneimittels ermittelt werden. Als Parameter zur Erhebung der klinischen Wirksamkeit («benefit») von onkologischen Krankheiten wird von der European Medicines Agency (EMA) das Gesamtüberleben (overall survival (OS)) und das progressionsbzw. krankheitsfreie Überleben (progression-free survival (PFS) bzw. disease-free survival (DFS)) empfohlen. In Zulassungsstudien wurden in der Vergangenheit jedoch häufig Surrogatendpunkte gewählt, anhand derer ein patientenrelevanter Nutzen nicht nachgewiesen werden kann, wie z.B. Tumorprogress oder Ansprechrate [10, 16, 18]. So ergab beispielsweise eine Analyse von 48 Studien zu onkologischen Arzneimitteln, die der EMA zwischen 1995 und 2004 für die Zulassung von 14 Wirkstoffen in 27 Indikationen vorgelegt wurden, dass zur Beurteilung der Wirksamkeit nur 7\% der Studien das Gesamtüberleben als primären Endpunkt ausgewertet hatten, dagegen $41 \%$ das progressionsfreie Überleben und $48 \%$ die Ansprechrate [16]. Die Arzneimittel waren häufig aufgrund kleiner, einarmiger Studien zugelassen worden, die keine verlässliche Einschätzung von Wirksamkeit und Toxizität zuließen. 
Aus anderen Untersuchungen ergibt sich Anhalt für weitere Defizite von Zulassungsstudien. So basiert die Beurteilung der Wirksamkeit neuer Arzneimittel, darunter auch zahlreiche onkologische Wirkstoffe, nur bei etwa der Hälfte der Zulassungen der amerikanischen (Food and Drug Administration (FDA)) bzw. europäischen (EMA) Zulassungsbehörde auf einer Studie mit einem aktiven Wirkstoff im Vergleichsarm [14]. Darüber hinaus werden wichtige Parameter für die Bewertung des Patientennutzens (z.B. Verbesserung der Lebensqualität, Symptomkontrolle) in Zulassungsstudien häufig nicht adäquat untersucht und Studien nach Zwischenanalysen, die zu diesem Zeitpunkt einen Nutzen für das Arzneimittel zeigen, vorzeitig abgebrochen, obwohl dies zu einer Überschätzung der Wirksamkeit und zur unzureichenden Bewertung der Sicherheit des Arzneimittels führen kann ([10, 16, 19-22], Übersicht in [18]).

Eine aktuelle Auswertung der zwischen 1. Januar 2009 und 25. August 2010 erschienenen Bewertungsberichte der EMA (European Assessment Report (EPAR)) zu 15 neuen onkologischen Wirkstoffen für 16 Indikationen hinsichtlich Studiendesign und Endpunkten [18] belegt, dass sich die Qualität der Zulassungsstudien in verschiedener Hinsicht verbessert zu haben scheint. Insgesamt wurden 18 entscheidende klinische Studien (pivotal trials) für die Zulassung bzw. Zulassungserweiterung vorgelegt, die zum Teil durch weitere Studien gestützt wurden ([18], unveröffentlichte Ergebnisse). In den meisten dieser Studien wurden die von der EMA empfohlenen Endpunkte Überleben $(\mathrm{n}=5)$ bzw. progressionsfreies Überleben $(n=9)$ verwendet, und mit einer Ausnahme handelte es sich um offene oder doppelt verblindete, randomisierte, kontrollierte Studien (RCT) mit einem Vergleichsarm, der allerdings in 7 Studien ein Placebo und nur in 4 Studien eine Standardtherapie enthielt. Auch hinsichtlich der Beachtung der Richtlinien für Good Clinical Practice (GCP) fanden sich weiterhin Qualitätsdefizite (z.B. Zugang zu den Studiendaten vom Sponsor verwehrt bzw. Liste zur Randomisierung nicht mehr verfügbar).

Neben der Kritik an der Qualität klinischer Zulassungsstudien sind systematische Verzerrungen (Bias) bei der Veröffentlichung von Studiendaten als weiterer limitierender Faktor für die Nutzen-Risiken-Bewertung zu benennen. So konnten kanadische Onkologen im Rahmen einer Analyse von RCTs hinsichtlich der chemotherapeutischen Behandlung von Mamma-, kolorektalen oder nicht kleinzelligen Bronchialkarzinomen, die zwischen 1975 und 2004 in 6 führenden medizinischen Fachzeitschriften veröffentlicht wurden, eine deutliche Beziehung zwischen Bevorzugung des experimentellen Arms und Sponsoring durch die pharmazeutische Industrie («sponsorship bias») zeigen [22]. Bias entsteht weiterhin dadurch, dass Studien mit positiven und signifikanten Ergebnissen eine größere Publikationschance haben als Studien mit negativen und nicht signifikanten Resultaten. Nur bei Kenntnis und Bewertung aller Studienergebnisse ist aber beispielsweise im Rahmen von systematischen Übersichts- arbeiten und Metaanalysen eine wissenschaftlich fundierte Nutzenbewertung neuer Arzneimittel möglich [23].

Zusammenfassend bewirken die systematischen Mängel in Phase-II- und -III-Studien vor Zulassung, dass die von den Zulassungsbehörden zu beurteilende Frage nach dem Wirksamkeit/Risiko-Verhältnis in einer gut charakterisierten, für den medizinischen Alltag relevanten Zielpopulation häufig nicht beantwortet werden kann.

Eine Verbesserung der Datenlage zur Bewertung des Nutzens und der Kosten-Nutzen-Relation neu zugelassener Arzneimittel in der Onkologie ist für eine rationale Verordnung kostenintensiver Wirkstoffe in der Onkologie dringend erforderlich. Dafür müssen zunächst die oben genannten Defizite in den klinischen Studien vor der Zulassung weiter abgebaut bzw. die heute gültigen Anforderungen an die Zulassung strenger beachtet werden. Darüber hinaus ist eine verstärkte Durchführung unabhängig von der Industrie initiierter, nichtkommerzieller klinischer Studien auch in der Onkologie unabdingbar $[17,24,25]$. Als problematisch ist hier allerdings die aktuelle Situation der nichtkommerziellen klinischen Forschung in Deutschland zu bewerten; dies insbesondere wegen der Schwierigkeiten bei der Finanzierung klinischer Studien, die nach Inkrafttreten der 12. Novelle des Arzneimittelgesetzes (AMG) deutlich gestiegenen Anforderungen an die Qualität des Designs und die Durchführung Rechnung tragen müssen [24, 25]. Neben einer ausreichenden öffentlichen Finanzierung wissenschaftsinitiierter, nichtkommerzieller klinischer Forschung stellt die organisatorische Bündelung dieser Aktivitäten beispielsweise im Sinne des Aufbaus sogenannter Clinical Trial Units, wie sie in Großbritannien beim Medical Research Council und in den USA beim National Cancer Institute angesiedelt sind, eine weitere Herausforderung dar. Dabei sollten die kürzlich vom Institute of Medicine (IOM) in den USA veröffentlichten Empfehlungen zur Verbesserung der klinischen Studien in der Onkologie beachtet werden [26]. Schließlich erscheinen Strategien zur Förderung der in der klinischen Forschung tätigen Nachwuchswissenschaftler wünschenswert, um die aktuell auch im Vergleich zur Grundlagenforschung wenig attraktiven Rahmenbedingungen für diesen Bereich zu verbessern [24].

\section{Strategien zur Implementierung von Nutzen- bzw. Nutzen-Risiken-Bewertungen}

Im Folgenden werden Strategien vorgestellt, mithilfe derer sowohl die Qualität wissenschaftlicher Daten als Grundlage für die Nutzen-Risiko-Bewertung zum Zeitpunkt der Zulassung als auch die Evidenzgenerierung bezüglich neuer Wirkstoffe nach ihrer Zulassung verbessert werden kann. In diesem Zusammenhang wird kurz auch auf die aktuelle Diskussion über den Gesetzesentwurf der Fraktionen der CDU/CSU und FDP zur Neuordnung des Arzneimittelmarktes in der gesetzlichen Krankenversicherung (Arzneimittelmarktneuordnungsgesetz (AMNOG)) eingegangen [27]. 


\section{Transparenz aller Studiendaten}

Empirische Untersuchungen belegen, dass die im Rahmen von Zulassungsstudien erhobenen Daten nur unvollständig beziehungsweise im Fall von negativen Ergebnissen häufig gar nicht publiziert werden. Vor dem Hintergrund dieses Publikationsbias und dem daraus resultierenden systematischen Fehler in der Nutzen-Risiko-Bewertung neuer Arzneimittel sollte gewährleistet werden, dass sämtliche klinische Studien einschließlich ihrer Ergebnisse und Studienprotokolle registriert und für die mit der Nutzenbewertung betrauten Institutionen zugänglich gemacht werden [28]. Die im Rahmen der Änderung des AMG geplante gesetzliche Verpflichtung zur Veröffentlichung der Ergebnisse konfirmatorischer klinischer Prüfungen ( $\$ 42 \mathrm{~b})$ sollte jedoch auch die Studienprotokolle umfassen $[29,30]$.

Horizon Scanning-System zur Früherkennung und Bewertung von neuen medikamentösen Therapiekonzepten

Die Entwicklung eines nationalen Scanning-Systems für die frühe Identifizierung und Evaluierung neuer Arzneimitteltherapien ermöglicht eine vorläufige Bewertung von Arzneimitteln, bevor sie in die Routinebehandlung eingeführt werden. Sogenannte Horizon Scanning-Systeme zur Früherkennung und Bewertung von neuen Arzneimitteln bzw. medikamentösen Therapiekonzepten sind heute bereits in verschiedenen europäischen Ländern eingeführt und dort vorwiegend bei Health Technology Assessment (HTA)-Organisationen angesiedelt (Übersicht in [31]). Im Fokus solcher Früherkennungssysteme stehen neue Arzneimittel bzw. neue Anwendungsgebiete vorhandener Arzneimittel, bei denen in naher Zukunft die Marktzulassung bzw. Zulassungserweiterung zu erwarten ist und die deshalb einer raschen Bewertung ihres therapeutischen (Zusatz-)Nutzens und ökonomischer (Budget-)Auswirkungen unterzogen werden sollen. Das Horizon Scanning beginnt $\mathrm{zu}$ unterschiedlichen Zeitpunkten (z.B. 6-36 Monate) vor der Zulassung und nutzt unterschiedliche Datenquellen (z.B. vom pharmazeutischen Hersteller, von den Zulassungsbehörden, wissenschaftliche Publikationen, Kongresspräsentationen). Wesentliches Ziel des Horizon Scannings ist die Bereitstellung unabhängiger Informationen bei Markteintritt, die bei der Planung eines optimierten Einsatzes bzw. Entscheidungen über die Aufnahme neuer Arzneimittel in den Leistungskatalog herangezogen werden können.

\section{Unabhängige klinische Studien nach Arzneimittelzulassung} Ziel von Post-Zulassungsstudien ist die Beantwortung der offenen, für die medikamentöse Versorgung der onkologischen Patienten relevanten Fragen. Dies gilt für alle neu zugelassenen Wirkstoffe, auch für die in der Onkologie rasch zunehmende Zahl der Wirkstoffe zur Behandlung seltener Erkrankungen (orphan drugs), für die zum Zeitpunkt der Zulassung bislang regelmäßig kein Nachweis über einen therapeutischen Zusatznutzen erfolgt ist [32]. Die im Rahmen des AMNOG geplante Ausnahmeregelung - nicht erforderlicher Nutzennachweis für orphan drugs - geht von falschen Annahmen aus (medizinischer Zusatznutzen durch die Zulassung belegt, Fehlen therapeutisch gleichwertiger Alternativen) und gefährdet die Versorgung von Patienten mit Arzneimitteln, die hinsichtlich ihrer Nutzen und Risiken ausreichend geprüft sind [8]. Bei der Ausgestaltung entsprechender Post-Zulassungsstudien müssen die Bewertungsmaßstäbe (z.B. relevanter Nutzen, Zusatznutzen), die Studienpopulation (z.B. Einschluss älterer Patienten), das Design (z.B. «head-to-head»Vergleich) und die Endpunkte (z.B. Überlebenszeit, krankheitsbezogene Lebensqualität) so definiert werden, dass die zugelassene Substanz unter Alltagsbedingungen und mit Blick auf versorgungsrelevante Kriterien bewertet werden kann [14]. Die Identifizierung der für diese Studien relevanten Fragestellungen sowie Vorschläge zum Studiendesign sollten durch ein unabhängiges Expertengremium erfolgen. Neben Vertretern von onkologischen Fachgesellschaften sowie von den in der klinischen Forschung engagierten Studiengruppen sollte durch Einbeziehung der Arzneimittelkommission der deutschen Ärzteschaft sowie weiterer Gremien der Selbstverwaltung (unter anderem Kassenärztliche Bundesvereinigung, KBV, GKV/PKV, Deutsche Krankenhausgesellschaft) und von Patientenvertretern garantiert werden, dass die für die Entscheidungsfindung relevanten Interessen berücksichtigt sind. Die Umsetzung entsprechender klinischer Studien sollte durch die Anpassung der administrativen Auflagen (siehe aktuelle Auflagen der 12. AMG-Novelle) sowie die ErschlieBung neuer Finanzierungsmöglichkeiten (unter anderem Ausbau der Förderung klinischer Studien durch das Bundesministerium für Bildung und Forschung (BMBF) und die Deutsche Forschungsgemeinschaft (DFG)) unterstützt werden (Übersicht bei [24, 25]).

\section{Einführung neuer Behandlungsverfahren in die Versorgung} Voraussetzung für den Einschluss eines zugelassenen Arzneimittels in die Regelversorgung zu Lasten der GKV beziehungsweise PKV ist ein günstiges Verhältnis von Nutzen und Risiken unter Alltagsbedingungen. Bei offenen, versorgungsrelevanten Fragestellungen stellt die Beschränkung der durch die GKV finanzierten Anwendung dieser neuen Wirkstoffe in Kliniken und onkologischen Praxen, die sich an Post-Zulassungsstudien beteiligen, eine Möglichkeit dar, in einem vergleichsweise kurzen Zeitraum die für die Nutzen-RisikoBewertung erforderlichen Daten zu erheben und wissenschaftlich auszuwerten.

\section{Fazit}

Eine Verbesserung der wissenschaftlichen Datenlage zur Bewertung des Nutzens beziehungsweise des Nutzen/ Risiko-Verhältnisses ist für eine rationale Verordnung und die Priorisierung kostenintensiver Wirkstoffe in der Häma- 
tologie/Onkologie unverzichtbar. Dies setzt insbesondere voraus, dass Evidenzlücken, die häufig aus Defiziten in den klinischen Studien vor Zulassung resultieren, rasch abgebaut und industrieunabhängige, versorgungsrelevante klinische Studien unmittelbar nach Zulassung zur Beantwortung offener Fragen initiiert werden. Grundsätzlich sollten alle Wege der Evidenzgenerierung (unter anderem randomisierte kontrollierte Studien, nichtinterventionelle Studien, Register) und mögliche Datenlieferer (unter anderem Kliniken, ambulante Schwerpunktpraxen) sowie Sponsoren
(Industrie und industrieunabhängige Sponsoren) in Betracht gezogen werden, um so rasch wie möglich Evidenz über den Nutzen und das Risiko von neuen onkologischen Arzneimitteln zu generieren.

\section{Disclosure Statement}

Die Autoren erklären, dass kein Interessenkonflikt im Sinne der Richtlinien des International Committee of Medical Journal Editors besteht.

\section{Literatur}

1 Bach PB: Limits on Medicare's ability to control rising spending on cancer drugs. N Engl J Med 2009;360:626-633.

2 Drummond MF, Mason AR: European perspective on the costs and cost-effectiveness of cancer therapies. J Clin Oncol 2007;25:191-195.

$\checkmark 3$ Sulmasy DP: Cancer care, money, and the value of life: Whose justice? Which rationality? J Clin Oncol 2007;25:217-222.

4 DiMasi JA, Grabowski HG: Economics of new oncology drug development. J Clin Oncol 2007;25: 209-216.

$\checkmark 5$ Gudiksen M, Fleming E, Furstentahl L, Ma P What drives success for speciality pharmaceuticals? Nat Rev Drug Discov 2008;7:563-567.

6 McCabe C, Bergmann L, Bosanquet N, et al.: Market and patient access to new oncology products in Europe: a current, multidisciplinary perspective. Ann Oncol 2009;20:403-412.

7 Trusheim MR, Berndt ER, Douglas FL: Stratified medicine: strategic and economic implications of combining drugs and biomarkers. Nat Rev Drug Discov 2007:6:287-293.

8 Windeler J, Koch K, Lange S, Ludwig WD: Zu guter Letzt ist alles selten. Dtsch Arztebl 2010;42: A2032-A2034.

-9 Aggarwal S: Targeted cancer therapies. Nat Rev Drug Discov 2010;9:427-428.

10 Apolone G, Tafuri G, Trotta F, Garattini S: A new anti-cancer drug in the market: Good news for investors or for patients? Eur J Cancer 2008;44:17861788

11 Medical Professionalism Project: Medical professionalism in the new millennium: a physicians' charter. Lancet 2002;359:520-522.

12 Lange S: Bedeutung von klinischen Studien für die Entscheidungsfindung in den Gremien der Selbstverwaltung. Onkologie 2010;33(Suppl 7):30-34.

13 Glaeske G: Mehr Sicherheit und Gerechtigkeit bei Behandlungsentscheidungen. Kosten-Nutzen-Analysen im Gesundheitssystem unter besonderer Berücksichtigung der Onkologie. Onkologe 2008;14: 660-668.
14 Eichler HG, Bloechl-Daum B, Abadie E, Barnett D, König F, Pearson S: Relative efficacy of drugs: an emerging issue between regulatory agencies and third-party payers. Nat Rev Drug Discov 2010;9: 277-291.

15 Francke R, Hart D: Bewertungskriterien und -methoden nach SGB V. MedR 2008;26:2-24.

16 Apolone G, Joppi R, Bertele V, Garattini S: Ten years of marketing approvals of anticancer drugs in Europe: regulatory policy and guidance documents need to find a balance between different pressures. Br J Cancer 2005;93:505-509.

17 Löffler M, Brosteanu O: Qualitätssicherung durch wissenschaftsinitiierte klinische Studien unter onkologischen Versorgungsbedingungen. Onkologe 2008;14:1252-1259.

18 Ludwig WD, Schott G: Offene Fragen in der medikamentösen Tumortherapie mit neuen Wirkstoffen. Onkologie 2010;33(Suppl 7):6-10.

19 Joly F, Vardy J, Pintilie M, Tannock IF: Quality of life and/or symptom control in randomized clinical trials for patients with advanced cancer. Ann Oncol 2007:18:1935-1942.

20 Trotta F, Apolone G, Garattini S, Tafuri G: Stopping a trial early in oncology: for patients or industry? Ann Oncol 2008;19:1347-1353.

21 Van Luijn JCF, Gribnau FW, Leufkens HGM: Availability of comparative trials for the assessment of new medicines in the European Union at the moment of market authorization. Br J Pharmacol 2006;63:159-162.

22 Booth CM, Cescon DW, Wang L, et al.: Evolution of the randomized controlled trial in oncology over three decades. J Clin Oncol 2008;26:5458-5464.

23 Ross JS, Mulvey GK, Hines EM, et al.: Trial publication after registration in ClinicalTrials.gov: A cross-sectional analysis. PLoS Med 2009;6: e1000144.

24 Seufferlein T, Adler G: Klinische Forschung in Deutschland am Beispiel der Onkologie. Onkologie 2010;33(Suppl 7):1-5.
25 Bührlen B, Georgieff P, Vollmar HC: Stand und Bedingungen klinischer Forschung in Deutschland und im Vergleich zu anderen Ländern unter besonderer Berücksichtigung nichtkommerzieller Studien. Büro für Technikfolgen-Abschätzung beim Deutschen Bundestag. Januar 2010, Abschlussbericht Nr. 135.

26 Young RC: Cancer clinical trials - A chronic but curable crisis. N Engl J Med 2010;363:306-309.

27 Bundesministerium für Gesundheit: Entwurf eines Gesetzes zur Neuordnung des Arzneimittelmarktes in der gesetzlichen Krankenversicherung. www. bundesgesundheitsministerium.de/cln_151/ nn_1168248/SharedDocs/Downloads/DE/ Standardartikel/G/Glossar-Gesetze/amnog,template $I d=$ raw, property $=$ publicationFile.pdf/amnog.pdf Stand 03.11.2010.

28 Strech D: Zur Ethik einer restriktiven Regulierung der Studienregistrierung. Ethik Med 2010. DOI: 10.1007/s00481-010-0071-2.

29 Schott G, Pachl H, Limbach U, Gundert-Remy U, Ludwig WD, Lieb K: The financing of drug trials by pharmaceutical companies and its consequences. Part 1: a qualitative, systematic review of the literature on possible influences on the findings, protocols, and quality of drug trials. Dtsch Arztebl Int 2010;107:279-285.

30 Schott G, Pachl H, Limbach U, Gundert-Remy U, Lieb K, Ludwig WD: The financing of drug trials by pharmaceutical companies and its consequences. Part 2: a qualitative, systematic review of the literature on possible influences on the findings, protocols, and quality of drug trials. Dtsch Arztebl Int 2010;107:295-301.

31 Wettermark B, Godman B, Eriksson C, et al.: Einführung neuer Arzneimittel in europäische Gesundheitssysteme. G+G Wissenschaft 2010;3:24-34.

32 Joppi R, Bertele V, Garattini S: Orphan drug development is not taking off. Br J Clin Pharmacol 2009:5:494-502. 